\title{
A Technical Guide for Fiber Tract Dissection of the Internal Capsule
}

\author{
Marcos Devanir Silva DA COSTA¹, Vinícius Lopes BRAGA², Kaan YAGMURLU³ , Ricardo Silva CENTENO, \\ Sérgio CAVALHEIRO', Feres CHADDAD-NETO \\ ${ }^{1}$ Federal University of Sao Paulo, Department of Neurosurgery, Sao Paulo, Brazil \\ ${ }^{2}$ Federal University of Sao Paulo, Paulista School of Medicine, Sao Paulo, Brazil \\ ${ }^{3}$ Barrow Neurological Institute, St. Joseph's Hospital and Medical Center, Department of Neurosurgery, Phoenix, Arizona, USA
}

\section{ABSTRACT}

AIM: Dissection of white fibers is important in identifying detailed neuroanatomical relationships. With tractrography it is possible to transport and apply this knowledge in a practical way to treat many diseases involving the white matter.

MATERIAL and METHODS: The Klingler method, subsequently disseminated by Türe with slight modifications was used.

RESULTS: We review some historical aspects of white fibers and provide a guide for dissection of the internal capsule. The removal of gray matter allowed us to obtain a view of the white matter. We removed all U-shaped fibers to expose the insular cortex. The cortex of the insular lobe was removed, which exposed the extreme capsule. The removal of the claustrum exposed the external capsule, which covers the lentiform nucleus, specifically the putamen. During dissection, removing some fibers of the external capsule produced windows in which the putamen could be medially visualized. Since the internal capsule lies medial to the lentiform nucleus, it was necessary to remove the nucleus in order to expose the internal capsule. We identified five regions of the internal capsule: the anterior limb, genu, posterior limb, and sublenticular and retrolenticular parts. Finally, we determined that the fibers of the corona radiata condense into the internal capsule at the level of the superior border of the lentiform nucleus.

CONCLUSION: Knowledge gained with the cadaveric fiber dissection technique can be applied in microsurgical practice and can be used to evaluate the surgical treatment for different tumors and vascular malformations.

KEYWORDS: Fiber tracking, Internal capsule, Klingler's method, White matter

\section{INTRODUCTION}

$\mathrm{F}$ iber dissection, an old technique that has evolved throughout history, utilizes specific neuroanatomical features to demonstrate the organization of nerve bundles and fibers in the white matter of the brain $(1,8,9)$.

Andreas Vesalius (1514-1564) first distinguished white matter from gray matter by features of color and texture, with the former being white and firm while the latter was described as yellowish and soft. One hundred years later, Marcello Malpighi (1628-1686) boiled brains in water to yield white matter. Using a simple microscope, he was then able to track fiber bundles and demonstrate their organization. After Malpighi, Thomas Willis (1621-1675) developed a scraping technique that allowed him to demonstrate the arrangement of bundles and fibers in the white matter (9). Another $17^{\text {th }}$ century anatomist, Raymond Vieussens (1641-1715), described the dissection technique along with his anatomical findings (the pyramids, inferior olives, striate bodies, and centrum semiovale). Vieussens adopted the scraping method used by Willis, as well as the boiling technique developed by Malpighi. However, instead of boiling brains in water, Vieussens improved the approach by boiling nervous tissue in oil. In his work, "Neurographia Universalis," Vieussens also demonstrated the continuity of

Corresponding author: Vinícius Lopes BRAGA

E-mail: viniciuslopesbraga@live.com 
white matter through the corona radiata, internal capsule, and pyramidal tract $(8,9)$. Félix Vicq d'Azyr (1748-1794) contributed to the neuroanatomical history of this technique by hardening the brain in an alcohol solution, facilitating the dissection process (9).

All of these researchers played fundamental roles in providing the first descriptions of the brain's features and in the development of fiber dissection techniques. Though subsequent researchers described new aspects of the brain, no major advances were made until those of Josef Klingler (1888-1963). Klingler revolutionized brain study by freezing and thawing previously fixed brain tissue. The freezing processes increased the volume of water in the brain and allowed ice crystals to separate the rather compact structure of the white matter. This facilitated dissection, as it softened the fiber tract, which was then easier to remove $(1,8)$.

Although Klingler's methods were groundbreaking, his technique was used in few studies, and fiber dissection has long been forgotten, especially after the development of the microtome and histological techniques. However, Klingler's method of white matter dissection was revitalized after the impressive trials of Dr. Türe, who employed this technique under an operating microscope. Using the operating microscope, Türe et al. were able to describe the lateral aspect of the brain in detail (8).

At present, correlations between three-dimensional aspects of anatomical fibers and their functions can be elucidated by combining dissections with magnetic resonance imaging (MRI). In fact, image-guided surgeries using computed tomography (CT) and magnetic resonance (MR) are increasingly common. Combining these two approaches (i.e., radiology and white matter dissection) offers advantages over histological and microtome methodologies in revealing the clinical functions of fiber tracts $(3,4,7)$. Here, we provide an anatomical and technical guide for the fiber dissection of the internal capsule.

\section{MATERIAL and METHODS}

Klingler's method uses $5 \%$ formalin to fix the brain because this ensures that even the deepest brain structures will be fixed. Moreover, the level of fixation continues to improve the longer the brain is left in formalin. Currently, a duration of 2 or 3 months is used for this process. After dissection begins, the brain remains preserved in 5\% formalin between dissection sessions (1). The method described in this article follows Klingler's method, which is now the most commonly used. However, in compliance with Türe's method, we fixed brains using $10 \%$ formalin and then maintained them in 5\% formalin between dissection sessions.

All specimens used in the current study were fresh, removed from the body approximately 18 hours after death and sent to us by the public organization, Serviço de Verificação de Óbitos (SVO), Sao Paulo, Brazil. The study was performed in accordance with the ethical standards of the 1964 Declaration of Helsinki.
Formalin solution has preservative, disinfectant, and antiseptic properties. In order to avoid structural deformities, brains were suspended in $10 \%$ formalin solution by the basilar artery. Following the 2- to 3-month fixation period, brains were removed from formalin solution and washed in running water for 2 days. Brains were then placed in a container with pure water, and the arachnoid membrane, pia mater, arteries, and veins were carefully removed. Brains were then frozen in the container at -5 to $-10^{\circ} \mathrm{C}$ for 1 or 2 weeks. After 2 or 3 weeks, brains were thawed at ambient temperature, and dissection was then initiated (Figure 1). It should be noted that this technique has two disadvantages. First, freezing followed by thawing can cause fissures in the specimens. Second, because dissection of certain parts of the cerebrum will destroy other bundles, not all fibers in the same cerebrum can be studied.

The instruments used in fiber dissections were dissectors, scissors, anatomical clamps, toothed clamps, and wooden spatulas made from depressor sticks (Figure 2) (2). To fabricate the spatulas, a scalpel blade (number 10 or 11) was used to cut the depressor stick into three parts, and the two outer portions of the stick were discarded. The ends of the central portion were then cut to make them straight, and one of the ends was slenderized with the scalpel blade on both sides-superior and inferior - to make it sharp. The slenderized end allowed for the removal of fibers during dissection. Constructing our own wooden spatulas facilitated our dissections because it allowed us to customize our spatula dimensions. Moreover, these spatulas were able to penetrate between gyri so that we could detach gray matter from white matter (Figures 3, 4). When only white matter remained, dissection of the fiber bundles began.

\section{RESULTS}

First, the association fibers or "U-shaped fibers" were removed. These fibers interconnect neighboring cortical regions. At a deeper layer, the long association fibers, such as the uncinate fasciculus, cingulum, superior and inferior longitudinal fasciculi, and inferior fronto-occipital fasciculus, are encountered. All association fibers have their routes within the same hemisphere. During dissections, short arcuate (U-shaped) fibers were removed before long fibers, since

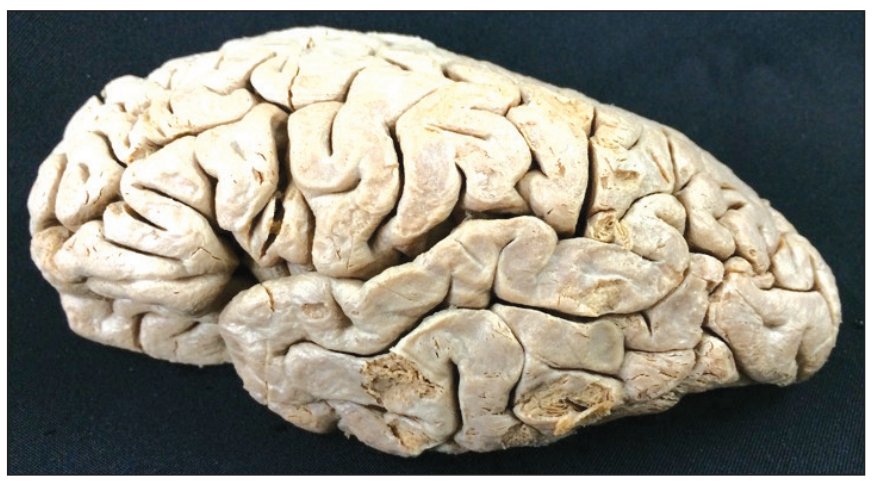

Figure 1: Aspect of a fixed and thawed hemisphere using Klingler's method. 


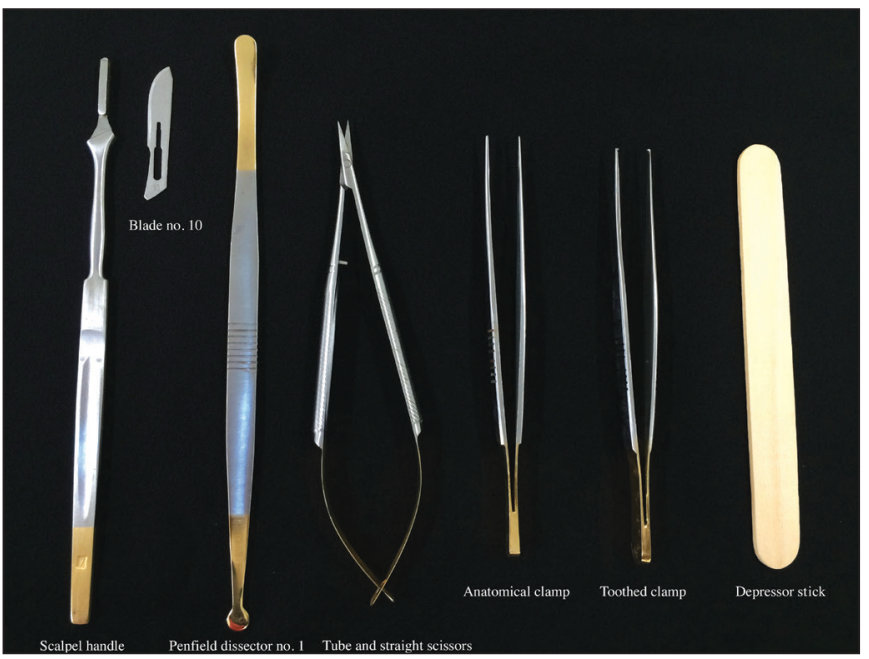

Figure 2: Instruments used in the fiber dissection: scalpel handle; blade no. 10; Penfield dissector no. 1; tube and straight scissors; anatomical clamp; toothed clamp; and depressor stick.

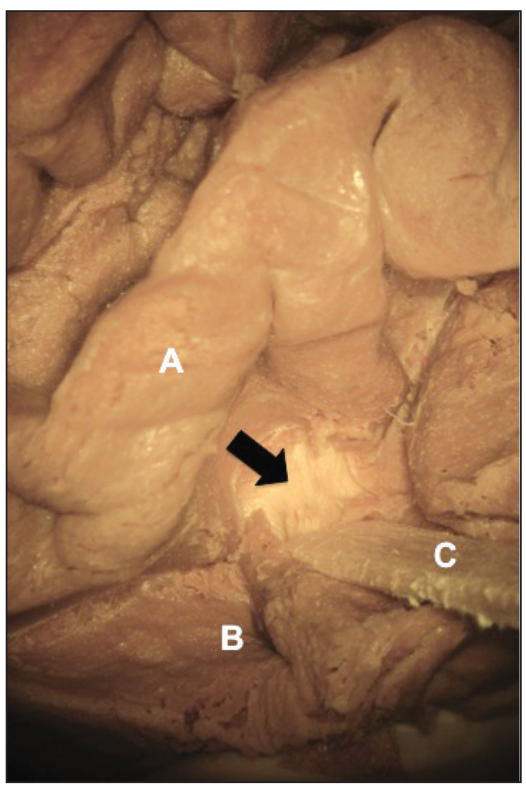

Figure 3: A magnified view showing easy detachment of gray from white matter using the wooden spatula in the base of the middle temporal gyrus.

A. Superior temporal gyrus. B. Middle temporal gyrus. C. Wooden spatula removing gray from white matter at the base of the gyrus. The black arrow points to the remaining white fibers.

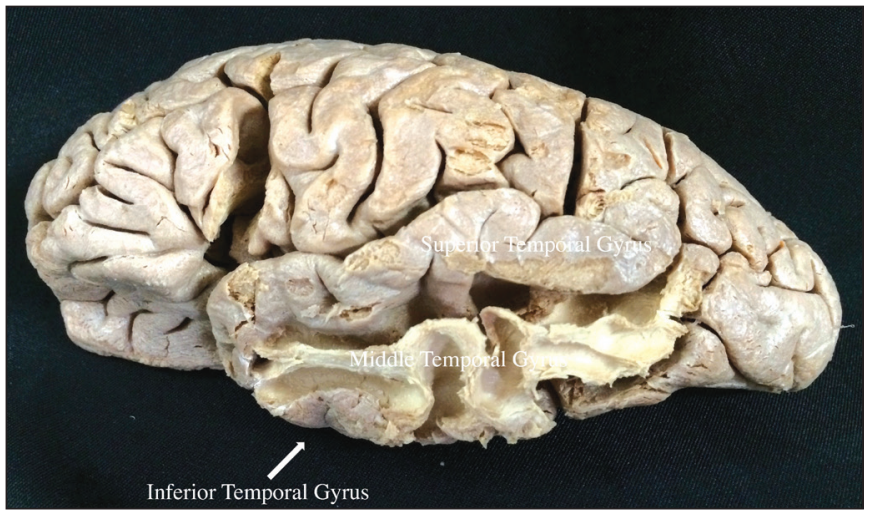

Figure 4: Magnified view of the result of gray matter removal around the middle temporal gyrus. short association fibers are externally located. Due to the extremely thin and faint nature of these fibers, dissectors, wooden spatulas, and sometimes even our hands helped in the removal process. Great care was taken during the procedure not to damage any fiber tract.

The commissural fibers interconnect the hemispheres by crossing the median plane. These fiber bundles include the corpus callosum (the major commissural fiber) and anterior and hippocampal commissures. Other projection fibers connect the cortex with the pons, medulla oblongata, and spinal cord in a rostrocaudal direction.

The entire process began with the removal of the gray matter (Figure 5), thereby exposing the white matter. Thereafter, we removed all U-shaped fibers from the superior, middle, and inferior temporal gyri and from the inferior and middle frontal gyri, as well as the inferior parietal lobule, to expose the insular cortex. The insular cortex served as an important landmark that allowed us to locate the portions of the internal capsule situated deep within the brain (Figure 6).

The cortex of the insular lobe was removed with wooden spatulas and dissectors, exposing the extreme capsule. Notably, the insula has a triangular shape with three corners:

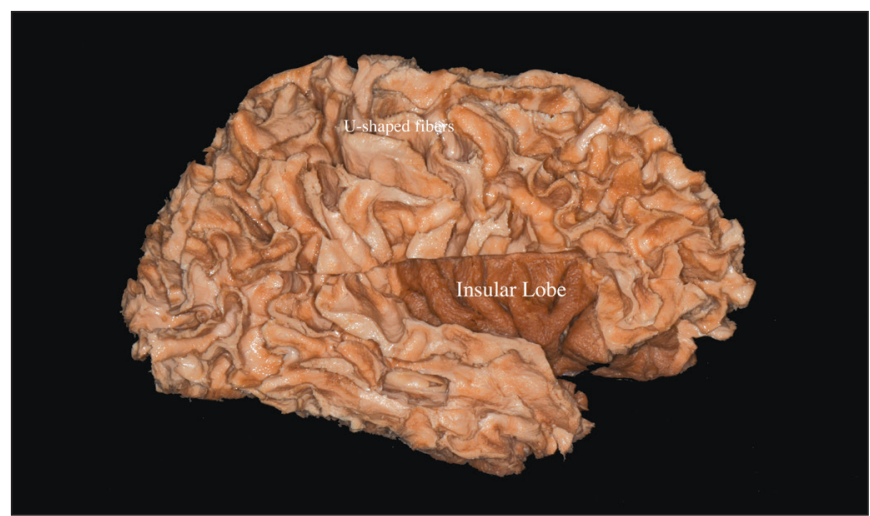

Figure 5: View of U-shaped fibers in the cerebral hemisphere without cortex, except in the insula, where the cortex remains.

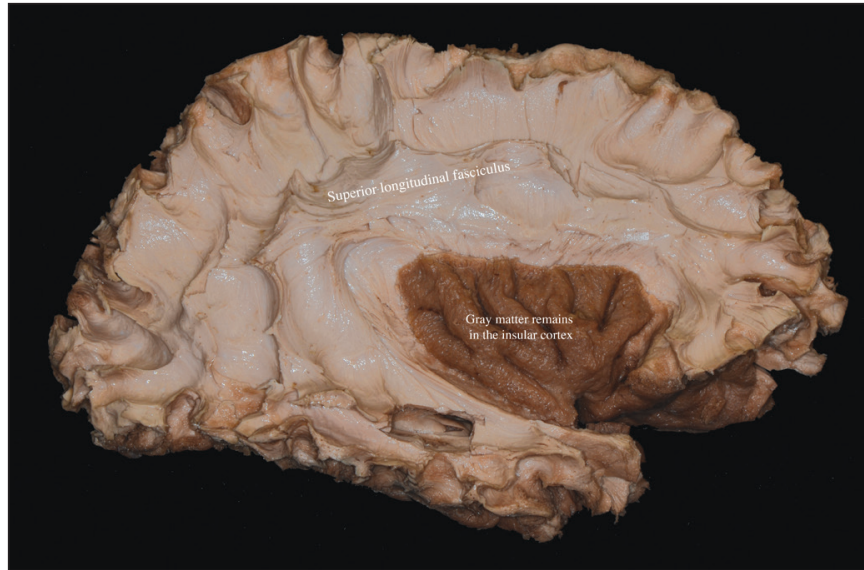

Figure 6: U-shaped fibers removed, exposing the superior longitudinal fasciculus, and insular cortex. 
a superior-anterior point (also known as the anterior insular point), an inferior-anterior point, and a posterior point (also known as the posterior insular point). The inferior-anterior and superior-anterior corners were covered by the pars triangularis of the inferior frontal gyrus, while the posterior corner was covered by the supramarginal gyrus (Figure 7).

Removal of the extreme capsule exposed the claustrum, a thin lamina of gray matter (Figure 8). The removal of the claustrum then exposed the external capsule, which covers the lentiform nucleus, composed of the putamen and globus pallidus. During dissection, the removal of some fibers of the external capsule produced windows exposing the putamen medially (Figures 9, 10). After removing the entire external capsule, we could observe the whole putamen and parts of the corona radiata, which terminates at the level of the superior border of the lentiform nucleus (Figure 11). The putamen, lateral medullary lamina, lateral globus pallidus, medial medullary lamina, and medial globus pallidus comprise the lentiform nucleus, which is located just lateral to the internal capsule.

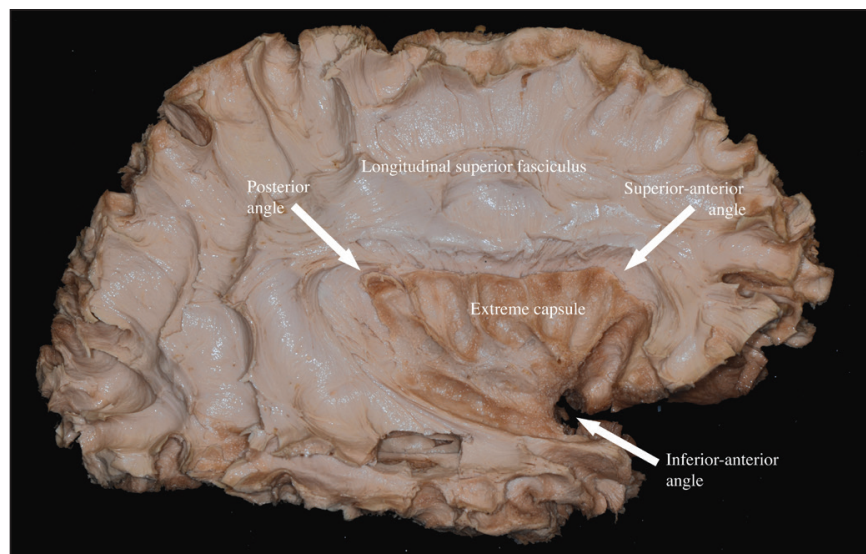

Figure 7: Figure illustrating the second step of the central core dissection, the removal of the insular gray matter exposes the extreme capsule (white matter of the insular lobe).

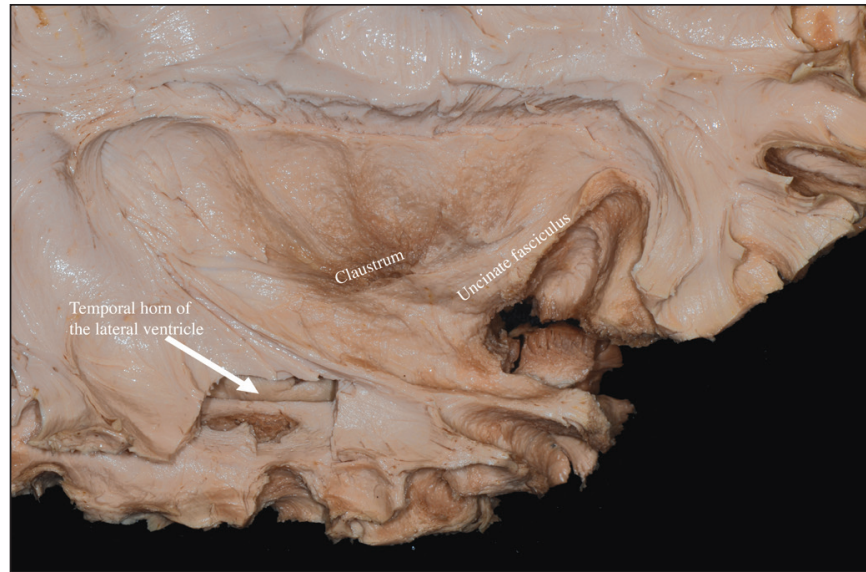

Figure 8: View of the claustrum after removal of the extreme capsule. This third step allows the visualization of the uncinate fasciculus and claustrum. Moreover the figure illustrates the temporal horn of the lateral ventricle by creating a window thru the lateral wall of the ventricle.

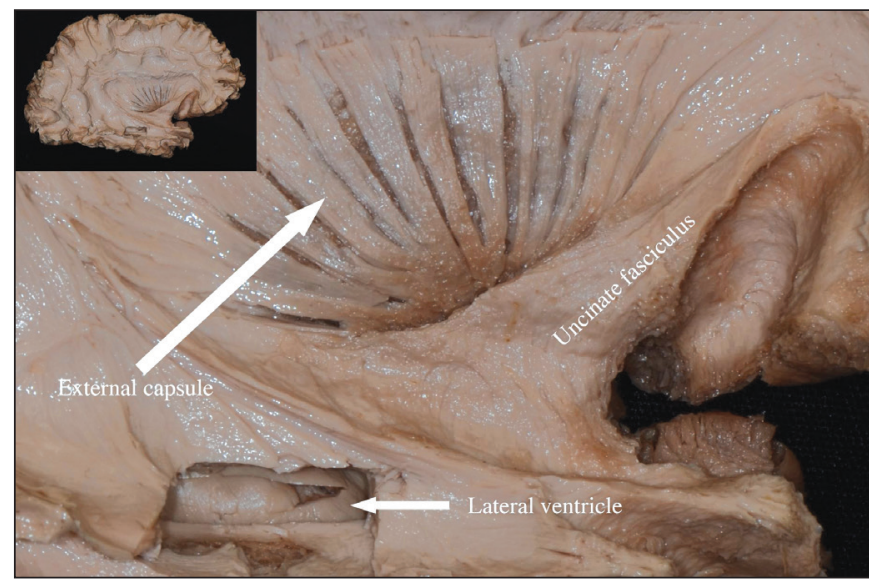

Figure 9: View after removal of the claustrum with the external capsule exposed. It is possible to identify the putamen between the fibers that comprise the external capsule.

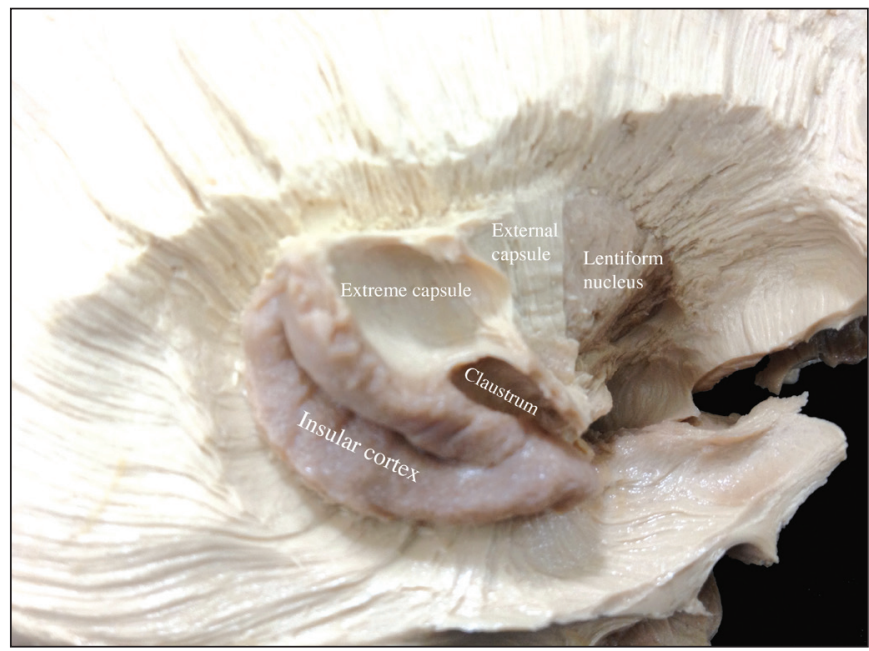

Figure 10: Magnified view of a multilayer dissection from lateral to medial: Insular cortex, Extreme capsule, Claustrum, External capsule, Lentiform nucleus.

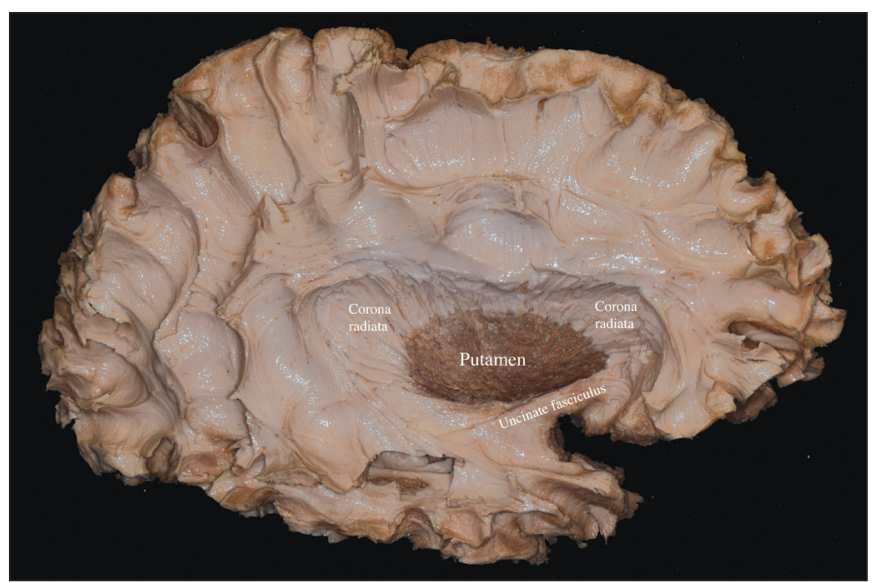

Figure 11: View of the putamen, the external part of the lentiform nucleus, and parts of the corona radiata. 
Since the internal capsule lies medial to the lentiform nucleus, it was necessary to remove this nucleus in order to expose the internal capsule (Figure 12). We identified five regions of the internal capsule: the anterior limb, genu, posterior limb, and sublenticular and retrolenticular parts. The anterior limb of the internal capsule separates the caudate nucleus medially and the putamen laterally. The anterior limb also carries fibers from the prefrontal and anterior cingulate cortices. The posterior limb of the internal capsule is located medial to the lentiform nucleus and lateral to the thalamus and carries thalamocortical and corticospinal fibers. These two limbs of the internal capsule curve in different directions, forming an arch. Between the two limbs, one can visualize the genu of the internal capsule, which travels medial to the foramen of Monro and lateral to the medial globus pallidus. The genu of the internal capsule contains corticothalamic, thalamocortical, and corticobulbar fibers. The sublenticular and retrolenticular parts of the internal capsule pass below and near the posterior edge of the lentiform nucleus, respectively. The sublenticular part of the internal capsule contains auditory radiation and optic radiation fibers, while the retrolenticular part carries some of the optic radiation fibers. Finally, we determined that the fibers of the corona radiata condense into the internal capsule at the level of the superior border of the lentiform nucleus (Figure 13). The medial limit of the internal capsule is the reticular nucleus of the thalamus.

\section{DISCUSSION}

Despite advances in radiological imaging techniques, no completely adequate technique for describing fiber pathways exists. The classic technique of cadaveric fiber dissection is still considered the best for neuroanatomical education of young neurosurgeons, and it is the gold standard among tractograpy techniques based on diffusion tensor imaging, MRI tractography, diffusion spectrum tractography, and autoradiographic techniques. Although the MRI technique can demonstrate fiber tracts in vivo, its limitation is the difficulty in determining the origin and termination of fiber pathways. The autoradiographic technique can be used only in experimental animals.

The knowledge of fiber tract anatomy is crucial for a better understanding of cognitive, psychiatric, and motor manifestations of white matter disorders, such as multiple sclerosis. In neurosurgery, understanding fiber pathway anatomy has become more even more important considering the plasticity of gray matter but not white matter, which indicates that any peri-operative damage to white matter causes irreversible deficits in patients (6). In preoperative surgical planning for removal of intra-axial lesions, the location and displacement of the important fiber pathways (the arcuate fasciculus, optic radiations, corticospinal tract, etc.) should be taken into the account for safer and more accurate surgery. Combining anatomical knowledge with preoperative MRI tractography provides excellent assessment and surgical planning based on each individual patient. For educational purposes, cadaveric fiber dissection under the operating microscope improves hand skill of the performer and provides

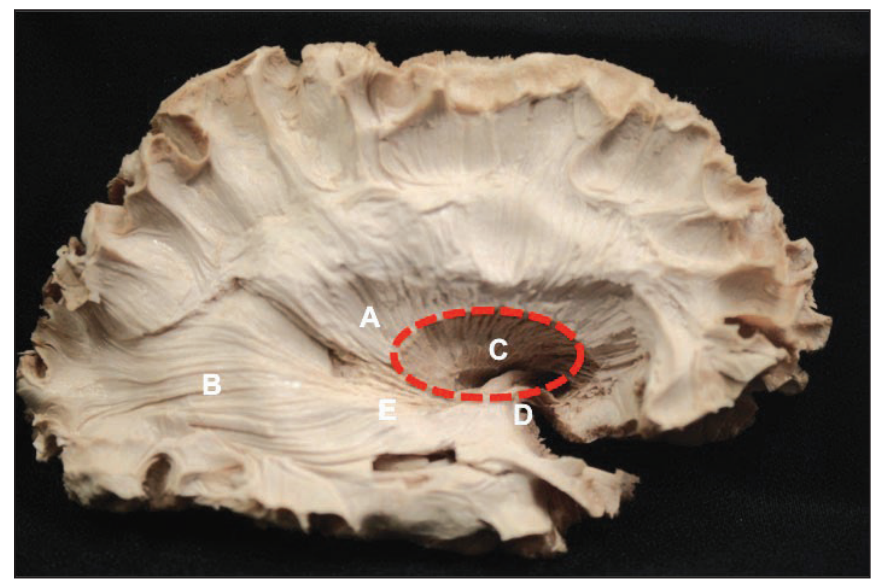

Figure 12: Notice that the putamen covers the internal capsule. The dashed red ellipse represents the area before it is occupied by the lentiform nucleus. Beneath the circle, it is easy to recognize the internal capsule. In this image, the superior and inferior fasciculus had already been removed to expose the entire corona radiata. A: Corona radiata. B: Optic radiation, part of the retrolenticular portion of the internal capsule. C: The blue circle represents the lentiform nucleus. D: Uncinate fasciculus. E: Sublenticular part of the internal capsule.

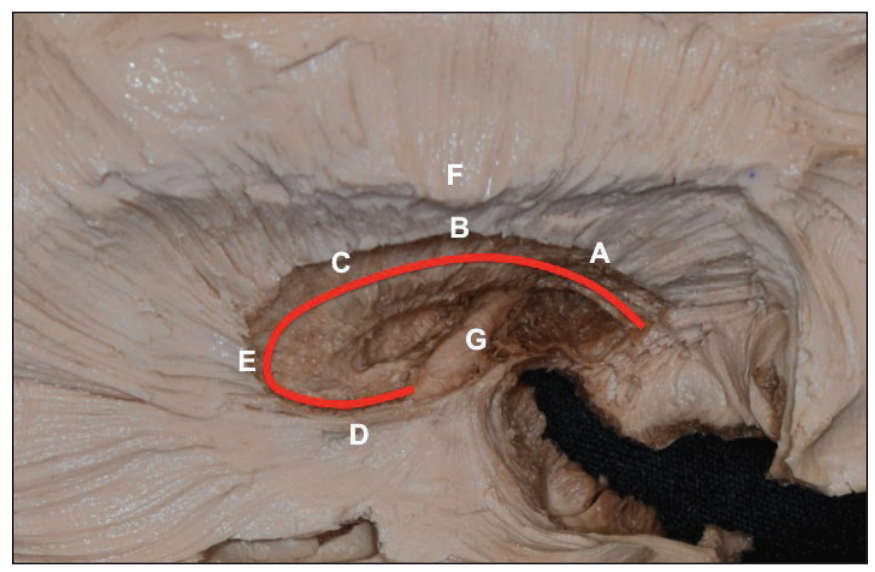

Figure 13: Magnified view of the internal capsule and it various parts. A: Anterior limb. B: Genu. C: Posterior limb D: Sublenticular part of the internal capsule. E: Retrolenticular part of the internal capsule. F: Corona radiata. G: Anterior commissure.

a three-dimensional understanding of complicated brain anatomy. To acquire this skill, the performer will spend hours or days in a microsurgery laboratory; the time-consuming nature of the cadaveric dissection technique is its only drawback. During the fiber dissection, the performer should follow only the fiber tracts during the dissection rather than what he/she want to see.

The technique described by Klingler is indispensable for white matter dissection and, in the current study, resulted in the separation of nearby fibers so that the internal capsule could be distinguished from structures lying in its path. Recent studies have improved upon Klingler's original method and added to our knowledge of the cerebrum by combining fiber 
dissection with MRI tractography (8). These seminal studies can help young neurosurgeons and medical students improve their knowledge of white fiber bundles, and the current report elucidates the pathway to the internal capsule.

After the decortication of gray matter, the site of initiation for removing U-shaped fibers is still controversial in fiber dissection practice. Previously, an initiation of dissection from the superior temporal sulcus was recommended to preserve the arcuate fasciculus (8). More recently, the subdivisions of the arcuate fasciculus in the inferior parietal lobe justify initiating the dissection from the inferior parietal lobe in order to obtain the segments of the arcuate fasciculus (10).

Regarding the position of the corticospinal tract in the internal capsule, there are two different standpoints. One claims that the corticospinal tract passes through the posterior half of the genu of the internal capsule; the other claims that the corticospinal tract passes through the anterior half of the posterior limb of the internal capsule. The surgical landmarks for the internal capsule are the superior-anterior corner of the insula and the anterior insular point for the anterior limb of the internal capsule, and the posterior corner and the posterior insular point for the posterior limb of the internal capsule $(5,8,10,11)$. The genu of the internal capsule corresponds to the same coronal level as the posterior short insular gyrus and foramen of Monro (11). The inferior limits of the parts of the internal capsule are the level of the anterior commissure for the anterior limb, the hypothalamus for the genu, and the lateral geniculate body for the posterior limb $(6,11)$.

\section{CONCLUSION}

Finally, knowledge gained with cadaveric fiber dissection technique can be applied in microsurgical practice and used to evaluate the surgical treatment for different tumors and vascular malformations.

\section{REFERENCES}

1. Agrawal A, Kapfhammer JP, Kress A, Wichers H, Deep A, Feindel W, Sonntag VK, Spetzler RF, Preul MC: Josef Klingler's models of white matter tracts: Influences on neuroanatomy, neurosurgery, and neuroimaging. Neurosurgery 69:238-252; discussion 252-234, 2011

2. Arnts $\mathrm{H}$, Kleinnijenhuis M, Kooloos JG, Schepens-Franke AN, van Cappellen van Walsum AM: Combining fiber dissection, plastination, and tractography for neuroanatomical education: Revealing the cerebellar nuclei and their white matter connections. Anat Sci Educ 7:47-55, 2014

3. Fernandez-Miranda JC, Rhoton AL Jr, Alvarez-Linera J, Kakizawa Y, Choi C, de Oliveira EP: Three-dimensional microsurgical and tractographic anatomy of the white matter of the human brain. Neurosurgery 62:989-1026; discussion 1026-1028, 2008

4. Kier EL, Staib LH, Davis LM, Bronen RA: Anatomic dissection tractography: A new method for precise MR localization of white matter tracts. AJNR Am J Neuroradiol 25:670-676, 2004

5. Ribas EC, Yagmurlu K, Wen HT, Rhoton AL Jr: Microsurgical anatomy of the inferior limiting insular sulcus and the temporal stem. J Neurosurg 122:1263-1273, 2015

6. Schmahmann J, Pandya D: Fiber Pathways of the Brain: Oxford University Press, 2009

7. Skadorwa T, Kunicki J, Nauman P, Ciszek B: Image-guided dissection of human white matter tracts as a new method of modern neuroanatomical training. Folia Morphol (Warsz) 68:135-139, 2009

8. Türe U, Yasargil MG, Friedman AH, Al-Mefty O: Fiber dissection technique: Lateral aspect of the brain. Neurosurgery 47:417426; discussion 426-417, 2000

9. Vergani F, Morris CM, Mitchell P, Duffau H: Raymond de Vieussens and his contribution to the study of white matter anatomy: Historical vignette. J Neurosurg 117:1070-1075, 2012

10. Yagmurlu K, Middlebrooks EH, Tanriover N, Rhoton AL Jr: Fiber tracts of the dorsal language stream in the human brain. J Neurosurg 124:1396-1405, 2016

11. Yagmurlu K, Vlasak AL, Rhoton AL Jr: Three-dimensional topographic fiber tract anatomy of the cerebrum. Neurosurgery 11 Suppl 2:274-305; discussion 305, 2015 